\title{
MORFOLOGIA FOLIAR E DENSIDADE DE HASTES DE HALODULE WRIGHTII (CYMODOCEACEAE), NO LITORAL DE ALAGOAS, BRASIL
}

Recebido em: julho/2013

Aceito em: agosto/2013

\author{
Cassio Rafael Campos de FRANÇA ${ }^{1}$ \\ Maria Elisa PITANGA ${ }^{2}$ \\ Maria Danise De Oliveira ALVES ${ }^{3}$ \\ Maria Elisabeth de ARAÚJO ${ }^{3}$ \\ Silmar Luiz SILVA ${ }^{4}$ \\ Karine Matos MAGALHÃES ${ }^{4}$
}

\section{RESUMO}

O objetivo do estudo foi caracterizar quatro populações de Halodule wrightii no litoral norte de Alagoas, nordeste brasileiro. Entre novembro de 2011 e janeiro de 2012 foram coletadas 12 amostras por praia, sendo quatro por mancha, sempre se evitando as bordas. Em laboratório foram determinadas a densidade, a biomassa, o comprimento e a largura de folhas, com as diferenças entre os pontos de coleta analisadas pelo teste t e ANOVA one-way. Constatou-se diferenças significativas nas características morfológicas entre as populações, sendo as plantas de Barra Grande as maiores e de Tatuamunha as mais largas. As densidades entre as praias não apresentaram diferenças significativas, contudo as plantas de Porto da Rua revelaram diferença significativa intrapopulacional. Foi confirmada a plasticidade morfológica dentro e entre as populações de $H$. wrightii sendo sugeridos novos estudos que incluam diferentes variáveis ambientais de qualidade de água.

Palavras chave: Capim agulha; morfologia; Atlântico Tropical.

\section{ABSTRACT}

The aim of this study was to characterize four Halodule wrightii population of Alagoas shore, northeastern Brazil. Between November 2011 and January 2012, twelve samples were collected at each site, distributed in four samples per meadow, always trying to avoid the borders. In the laboratory shoot density, biomass (per core) and leave measured (length and width) were determinate and differences between the sites were tested by the test $t$ and one-way ANOVA analyses. There were no significant differences in morphology between populations, the plants were the largest of Barra Grande and the widest Tatuamunha. The densities of the beaches showed no significant differences, however plants of Porto da Rua revealed significant intra population differences. Morphological plasticity within and between populations of H. wrightii was confirmed what suggests the need of further studies including water quality

Key-word: Shoal grass; morphology; Tropical Atlantic.

\section{INTRODUÇÃO}

As angiospermas marinhas, por ocorrerem em ambientes costeiros dinâmicos e com diferentes gradientes de luz (STEWARD et al., 2005), sedimento (HAUXWELL et al., 2003; $\mathrm{KOCH}$ et al., 2007), salinidade (LIRMAN; CROPPER, 2003; $\mathrm{KOCH}$ et al., 2007), nutrientes e temperatura (FERDIE; FOURQUREAN, 2004; HUGHES et al., 2004), apresentam plasticidade

1 - Especialização em Oceanografia, Universidade Federal de Pernambuco.

2 - Programa de Pós-graduação em Oceanografia, Universidade Federal de Pernambuco

3 Departamento de Biologia, Universidade Federal Rural de Pernambuco 
FRANÇA, C. R. C. de et. al. Morfologia foliar e densidade de hastes de Halodule wrightii (Cymodoceaceae), no litoral de Alagoas, Brasil.

morfológica como forma de adaptação, permitindo-lhes uma melhor absorção dos recursos disponíveis no ambiente e sua sobrevivência (HOUSTON; MCNAMARA, 1992; PHILLIP, 1988). Fatores como luz, sedimento e nutrientes podem alterar algumas características morfológicas das plantas, tais como densidade de hastes, comprimento e largura foliar, além de influenciar a quantidade de epífitas e o número de plantas por unidade de área no prado (HEMMINGA; DUARTE, 2000; RUIZ; ROMERO, 2003).

A biorregião do Atlântico Tropical apresenta uma ampla diversidade de angiospermas marinhas (SHORT et al., 2007) sendo cinco espécies citadas para o litoral brasileiro (OLIVEIRAFILHO et al., 1983; SHORT et al., 2007; BARROS et al., 2013). A espécie Halodule wrightii é mais frequente ao longo da costa brasileira, com distribuição entre os estados do Piauí e Paraná (OLIVEIRA-FILHO; PIRANI; GIULIETTI, 1983; SORDO et al., 2011), formando prados desde águas rasas até $10 \mathrm{~m}$ de profundidade (LABOREL-DEGUEN, 1963). Por constituir a espécie dominante e de maior ocorrência, diversos estudos já foram realizados no litoral do Brasil sobre sua distribuição e morfologia, como de Magalhães, Eskinazi-Leça e Moura Júnior (1997) em Pernambuco, Creed (1999) e Creed e Monteiro (2000) no Rio de Janeiro, Oliveira et al. (1997) em São Paulo e Sordo et al. (op. cit.) no Paraná. Contudo, ainda há lacunas a serem preenchidas com relação à sua morfologia, padrões de distribuição e variações na biomassa, principalmente para o nordeste do país.

Diferenças morfológicas entre e dentro de prados são comuns nas angiospermas marinhas (PHILLIPS, 1992; BANDEIRA, 2002). Para Halodule wrightii, espécie dominante, sustenta-se a tese de que as diferenças morfológicas entre as espécies do gênero são devido à plasticidade morfológica dessas plantas para se adaptarem às condições ambientais (PHILLIPS, 1992), profundidade e exposição ao ar (CREED, 1997; MAGALHÃES, 1997).

O presente estudo teve por objetivo localizar e descrever a variação na morfometria foliar e na biomassa e densidade de hastes em quatro populações de Halodule wrightii no litoral norte de Alagoas, sendo o primeiro estudo para os prados do Estado.

\section{Área de estudo}

\section{MATERIAL E MÉTODOS}

O litoral de Alagoas possui cerca $230 \mathrm{~km}$ de linha de costa e está localizado na região nordeste do Brasil (Figura 1). O litoral apresenta regime de marés semidiurnas, o clima caracterizado como quente e úmido (tropical e equatorial), do tipo As de acordo com a classificação de Koppen. Com duas estações definidas ao longo do ano, uma chuvosa (março a agosto) e outra de estiagem (setembro a fevereiro), controlado por massas quentes equatoriais e tropicais (CORREIA; SOVIERZOSKI, 2008).

Tabela 1 - Pontos de coleta de Halodule wrightii no litoral norte do estado de Alagoas, nordeste brasileiro.

\begin{tabular}{lccc}
\hline Ponto de coleta & Ambiente & $\begin{array}{c}\text { Distância média da } \\
\text { linha de praia (m) }\end{array}$ & $\begin{array}{c}\text { Profundidade } \\
\text { média (cm) }\end{array}$ \\
\hline Barra Grande & Marinho & 0,11 & 94,43 \\
Tatuamunha & Marinho & 0,26 & 17,66 \\
Porto da Rua & Marinho & 0,41 & 26,00 \\
Barra de Camaragibe & Estuarino & 0,31 & 23,66 \\
\hline
\end{tabular}

\section{Coleta e processamento dos dados}

As coletas das plantas e de sedimentos foram realizadas durante o período de baixa-mar (maré $\leq 0.4 \mathrm{~m}$ ), em maré de sizígia, entre novembro de 2011 e janeiro de 2012 . Em cada praia foram selecionadas as três manchas mais representativas, em termos de área de cobertura, onde foram retiradas quatro amostras, na região central da mancha, utilizando um tubo coletor com $9.8 \mathrm{~cm}$ de diâmetro ( $\mathrm{n}=12$ por praia). Em laboratório, as hastes de Halodule wrightii foram contadas para determinação da densidade de hastes (hastes. $\mathrm{m}^{-2}$ ), e a biomassa aérea e subterrânea (g.ps. $\mathrm{m}^{-2}$ ) obtidas conforme o método descrito por Duarte e Kirkman (2001). Antes da secagem das plantas, foram selecionadas dez folhas inteiras por amostra para medição do 
FRANÇA, C. R. C. de et. al. Morfologia foliar e densidade de hastes de Halodule wrightii (Cymodoceaceae), no litoral de Alagoas, Brasil.

comprimento $(\mathrm{cm})$ e largura $(\mathrm{mm})$, com o auxílio de régua e de microscópio com lente micrometrada, respectivamente.

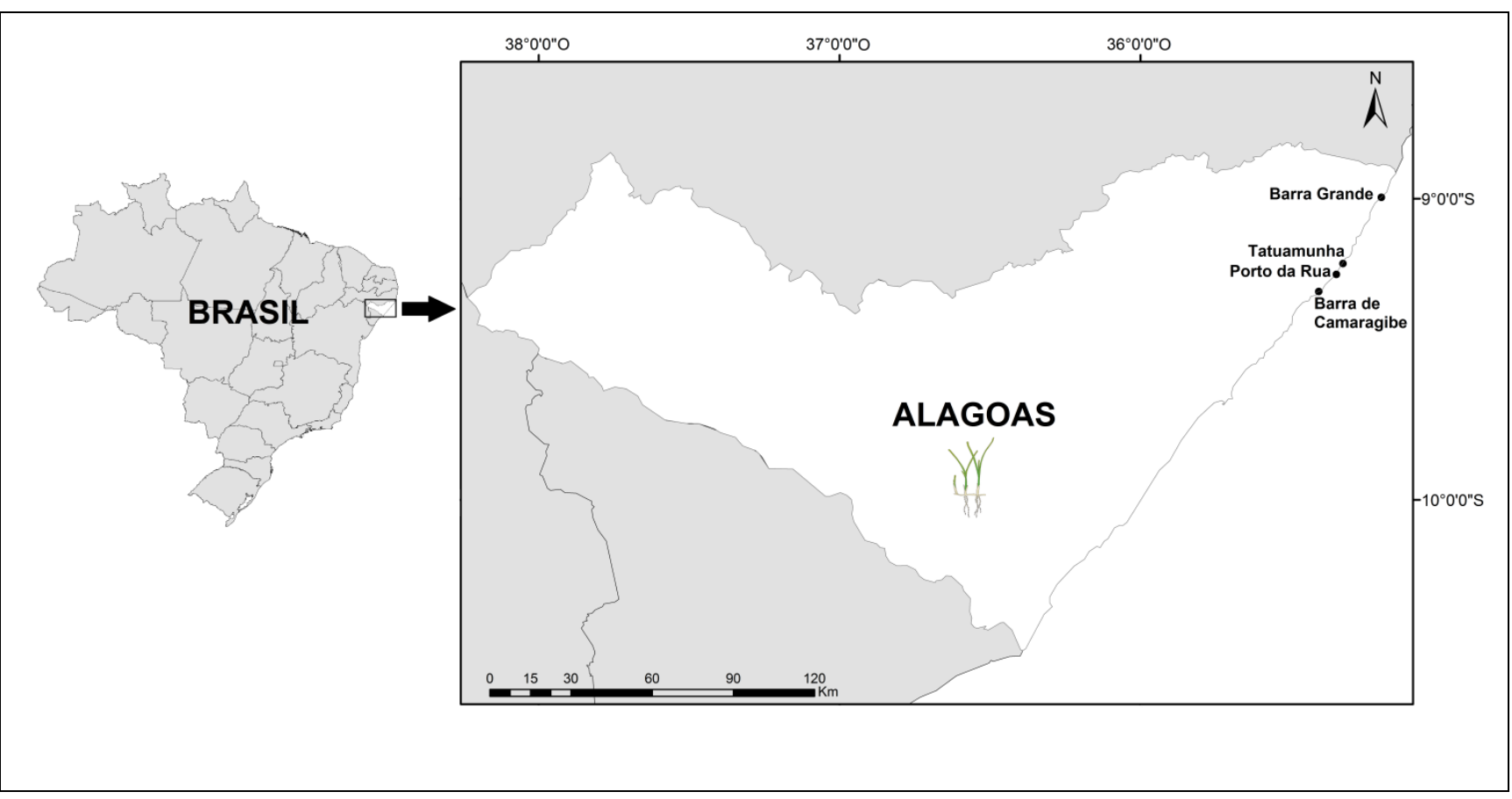

Figura 1 - Área de estudo localizada no litoral norte de Alagoas, nordeste do Brasil, com os pontos de amostragens de Halodule wrightii nas praias de Barra Grande, Tatuamunha, Porto da Rua e Barra de Camaragibe.

Amostras de sedimento foram coletadas em cada mancha (aproximadamente $15 \mathrm{~cm}$ de profundidade), na região central, com auxílio de um tubo coletor de $5 \mathrm{~cm}$ de diâmetro. Foram analisados os teores de carbonato de cálcio (LORING e RANTALA, 1992) e matéria orgânica (BYERS, MILLS e STEWART, 1978).

Para detectar diferenças significativas entre as características morfológicas e as áreas estudadas, foi aplicada a ANOVA One-Way, utilizando-se o Teste de Tukey para identificar possíveis diferenças entre as praias. $O$ valor de a aceito foi de 0.05 . Todos os testes foram realizados com o programa Statistica $8.0^{\circledR}$.

\section{RESULTADOS}

A densidade de hastes de Halodule wrightii variou entre 4510 hastes. $\mathrm{m}^{-2}$ (Tatuamunha) e 22815 hastes. $\mathrm{m}^{-2}$ (Barra de Camaragibe). Os menores valores médios foram registrados para Barra Grande $\left(9904 \pm 2484\right.$ hastes. $\left.\mathrm{m}^{-2}\right)$, enquanto os maiores valores de densidade média de hastes de $H$. wrightii foram registrados em Porto da Rua (12667 $\$ 3297$ hastes. $\mathrm{m}^{-2}$ ). Não foram observadas diferenças significativas entre as populações $(F=1,32 ; p=0,27)$ e dentro de cada população (Fig. 2, Tab. 2).

A biomassa total de Halodule wrightii variou entre 30,24 e $1220,98 \mathrm{~g} \mathrm{ps} \mathrm{m}^{-2}$ em Tatuamunha e Porto de Pedras, respectivamente (Figura 3). Constatou-se diferenças apenas entre as populações de Barra Grande e Porto da Rua ( $F=3.32 ; p=0.022)$. A biomassa aérea de Halodule wrightii variou entre 0,80 e $270,80 \mathrm{~g} \mathrm{ps} \mathrm{m}^{-2}$ para folhas em Porto de Pedras e Barra Grande, respectivamente, e 27,86 e $1208,38 \mathrm{~g} \mathrm{ps} \mathrm{m}^{-2}$ para parte subterrânea em Tatuamunha e Porto de Pedras, respectivamente (Tabela 2; Figura 4). 
Tabela 2 - Valores mínimos, máximos e médios registrados para as quatro populações de Halodule wrightii no litoral de Alagoas (Brasil), entre novembro de 2011 e janeiro de 2012. Onde: DENS=Densidade de hastes; BA=Biomassa aérea; BS=Biomassa subterrânea; Comp. Foliar=Comprimento foliar; $\mathrm{MO}=$ matéria orgânica; $\mathrm{CaCo}^{3}=$ Carbonato de cálcio.

\begin{tabular}{|c|c|c|c|c|c|c|c|}
\hline Locais de coletas & $\begin{array}{c}\text { DENS } \\
\left(\text { hastes.m }^{-2}\right)\end{array}$ & $\begin{array}{c}\text { BA } \\
\left(\text { g.ps.m }{ }^{-2}\right)\end{array}$ & $\begin{array}{c}\text { BS } \\
\left(\text { g.ps.m }{ }^{-2}\right)\end{array}$ & $\begin{array}{l}\text { Comp Foliar } \\
\qquad(\mathrm{cm}\end{array}$ & $\begin{array}{l}\text { Largura Foliar } \\
\qquad(\mathrm{mm})\end{array}$ & $\begin{array}{l}\text { MO } \\
(\%)\end{array}$ & $\begin{array}{c}\mathrm{CaCO}_{3} \\
(\%)\end{array}$ \\
\hline Barra Grande & $\begin{array}{l}5836-13132 \\
(9904 \pm 717)\end{array}$ & $\begin{array}{c}14,59-217,80 \\
(94,63 \pm 16,09)\end{array}$ & $\begin{array}{c}141,00-416,24 \\
(262,97 \pm 22,93)\end{array}$ & $\begin{array}{c}6,08-18,63 \\
(15,28 \pm 1,04)\end{array}$ & $\begin{array}{c}0,57-0,92 \\
(0,79 \pm 0,09)\end{array}$ & $3.00 \pm 0.00$ & $85.63 \pm 1.27$ \\
\hline Tatuamunha & $\begin{array}{c}4510-19233 \\
(10822 \pm 1146)\end{array}$ & $\begin{array}{c}2,39-34,35 \\
(12,19 \pm 2,89)\end{array}$ & $\begin{array}{c}27,86-262,63 \\
(148,56 \pm 21,11)\end{array}$ & $\begin{array}{c}3,95-7,89 \\
(6,14 \pm 0,33)\end{array}$ & $\begin{array}{c}0,62-0,96 \\
(0,77 \pm 0,11)\end{array}$ & $5.33 \pm 1.09$ & $76.87 \pm 4.65$ \\
\hline Porto da Rua & $\begin{array}{c}7561-16978 \\
(12667 \pm 952)\end{array}$ & $\begin{array}{c}0,80-29,98 \\
(13,45 \pm 2,65)\end{array}$ & $\begin{array}{c}34,49-1208,38 \\
(317,68 \pm 100,90)\end{array}$ & $\begin{array}{c}3,79-8,86 \\
(6,39 \pm 0,49)\end{array}$ & $\begin{array}{c}0,38-0,80 \\
(0,59 \pm 0,15)\end{array}$ & $2.67 \pm 0.60$ & $90.03 \pm 1.57$ \\
\hline $\begin{array}{l}\text { Barra de } \\
\text { Camaragibe }\end{array}$ & $\begin{array}{c}5040-22815 \\
(9992 \pm 1388)\end{array}$ & $\begin{array}{c}3,18-17,77 \\
(11,65 \pm 1,59)\end{array}$ & $\begin{array}{c}37,14-437,72 \\
(143,92 \pm 33,20)\end{array}$ & $\begin{array}{c}6,41-11,10 \\
(8,45 \pm 0,39)\end{array}$ & $\begin{array}{c}0,58-0,77 \\
(0,69 \pm 0,06)\end{array}$ & $2.00 \pm 0.29$ & $41.50 \pm 14.24$ \\
\hline
\end{tabular}

1 - Especialização em Oceanografia, Universidade Federal de Pernambuco.

2 - Programa de Pós-graduação em Oceanografia, Universidade Federal de Pernambuco

3 Departamento de Biologia, Universidade Federal Rural de Pernambuco

Contato: cassiorcfranca@hotmail.com 


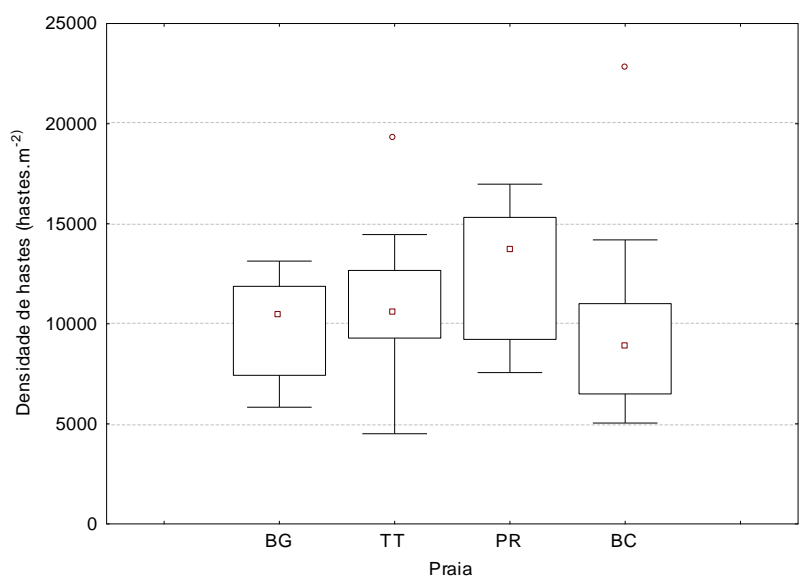

Figura 2 - Densidade foliar de Halodule wrightii nas praias estudadas de Alagoas, nordeste brasileiro, entre novembro de 2011 e janeiro de 2012. BC - Barra de Camaragibe $(F=2,21 ; p=0,16) ; \pi$ - Tatuamunha $(F=4,16 ; p=0,051) ; P R$ - Porto da Rua $(F=7,11 ; p=0,01) ; B G$ - Barra Grande $(F=0,55 ; p=0,59)$.

Mediana $=\square \quad 25 \%-75 \%=\square \quad$ Non-Outlier Range $=I \quad$ Outliers $=0$

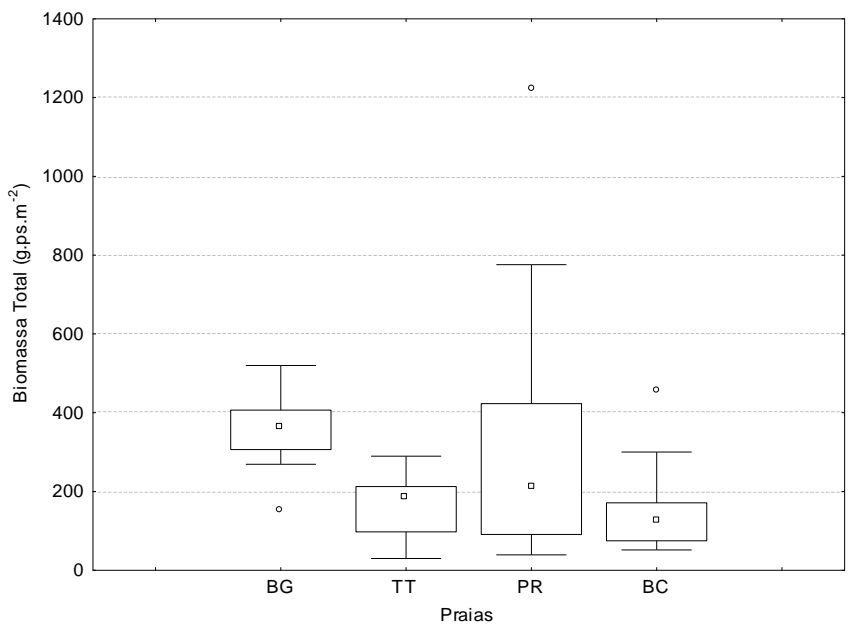

Figura 3 - Biomassa total (g.ps. $\mathrm{m}^{-2}$ ) das quatro populações de Halodule wrightii no litoral norte de Alagoas, nordeste brasileiro, entre novembro de 2011 e janeiro de 2012. BC Barra de Camaragibe; TT - Tatuamunha; PR - Porto da Rua; BG - Barra Grande. Mediana $=\square \quad 25 \%-75 \%=\square$ Non-Outlier Range $=\square \quad$ Outliers $=0$

Os resultados morfométricos evidenciaram os menores comprimentos foliares na praia de Porto de Pedras, enquanto a praia de Barra Grande apresentou os maiores com variação entre 3,57 e 18,63 (Tab. 2). Foram observadas diferenças significativas entre as praias pela ANOVA $(F=47,32 ; p \leq 0,05)$, diferença reforçada pelos resultados do teste de Tukey. Em relação à Barra Grande, os valores desse teste foram diferentes para todas as outras praias; Barra de Camaragibe $(F=10,99 ; p<0,01)$, Tatuamunha $(F=14,66 ; p<0,01)$ e Porto da Rua $(F=14,66$; $\mathrm{p}<0,01)$. Não foram observadas diferenças significativas dentro de cada praia (Fig. 4).

1 - Especialização em Oceanografia, Universidade Federal de Pernambuco.

2 - Programa de Pós-graduação em Oceanografia, Universidade Federal de Pernambuco 
FRANÇA, C. R. C. de et. al. Morfologia foliar e densidade de hastes de Halodule wrightii (Cymodoceaceae), no litoral de Alagoas, Brasil.

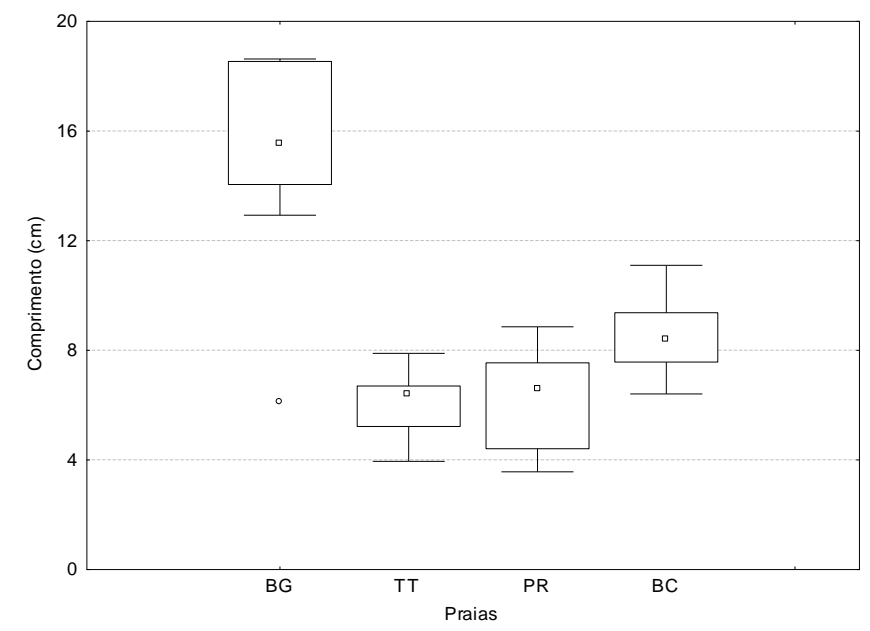

Figura 4 - Comprimento foliar de Halodule wrightii nas praias estudadas de Alagoas, nordeste brasileiro, entre novembro de 2011 e janeiro de 2012. BC - Barra de Camaragibe; TT - Tatuamunha; PR - Porto da Rua; BG - Barra Grande.

Mediana $=\square \quad 25 \%-75 \%=\square$ Non-Outlier Range $=\perp \quad$ Outliers $=0$

A largura das folhas variaram entre 0,38 e 0,96 cm para Porto de Pedra e Tatuamunha, respectivamente (Tab. 2). Os resultados revelaram diferenças significativas entre as praias $(F=8,98 ; p=0,0002)$, o que foi confirmado através do teste de Tukey no qual foi possível observar diferenças significativas entre Tatuamunha e Porto da Rua $(F=6,30 ; p<0,0001)$ e entre Porto da Rua e Barra Grande $(F=6,22 ; p<0,0001)$. Foram observadas, ainda, diferenças significativas entre as amostras das praias de Barra de Camaragibe e Porto da Rua (Fig. 5).

O teor médio de matéria orgânica foi considerado baixo, com valores médios mínimo de $2.00 \pm 0.29 \%$ (Barra de Camaragibe) e o máximo valor $5.33 \pm 1.09 \%$ (Tatuamunha). Foram verificadas diferenças significativas entre as praias $(F=4.34 ; p=0.04)$ tendo Tatuamunha apresentado a maior variação em relação às demais praias estudadas. Para o carbonato de cálcio o menor percentual médio $(41.50 \pm 14.24 \%)$ foi registrado para Barra de Camaragibe e o maior $(90,03 \pm 1.57 \%)$ para Porto da Rua. Foram encontradas diferenças significativas entre as praias $(F=4.72 ; p=0.04)$.

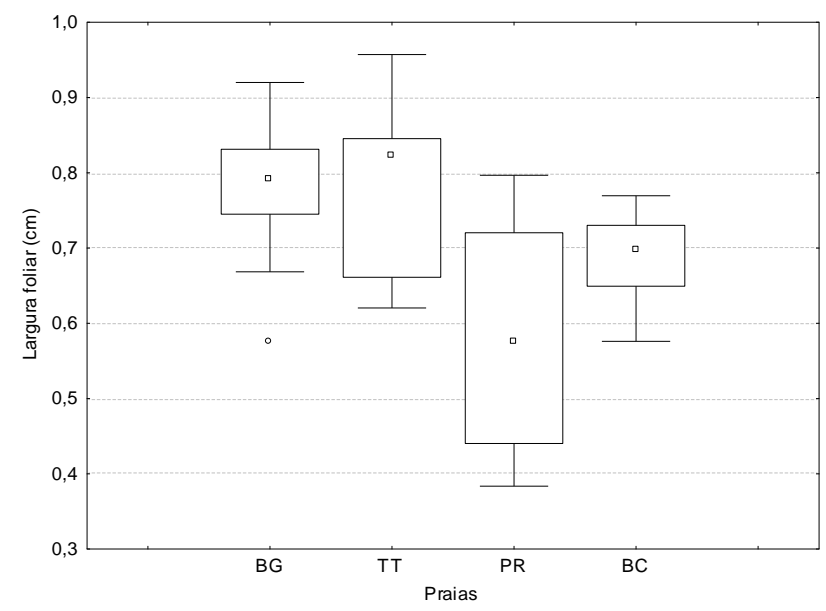

Figura 5 - Largura foliar de $H$. wrightii nas praias estudadas de Alagoas, nordeste brasileiro, entre novembro de 2011 e janeiro de 2012. BC - Barra de Camaragibe $(F=11,7$; $\mathrm{p}=0,003) ; \pi \mathrm{T}$ - Tatuamunha $(\mathrm{F}=1,01 ; \mathrm{p}=0,4) ; \mathrm{PR}$ - Porto da Rua $(\mathrm{F}=22,46$; $\mathrm{p}=0,0006)$; $\mathrm{BG}$ - Barra Grande $(\mathrm{F}=1,05 ; \mathrm{p}=0,39)$.

Mediana $=\square \quad 25 \%-75 \%=\square \quad$ Non-Outlier Range $=工 \quad$ Outliers $=0$ 
FRANÇA, C. R. C. de et. al. Morfologia foliar e densidade de hastes de Halodule wrightii (Cymodoceaceae), no litoral de Alagoas, Brasil.

\section{DISCUSSÃO}

Apesar das angiospermas marinhas serem frequentemente citadas por locais como alimento do peixe-boi marinho, o presente trabalho apresenta os primeiros dados ecológicos sobre esta espécie dentro do litoral alagoano da APA Costa dos Corais. Halodue wrightii exibiu diferenças morfológicas e estruturais que variaram de acordo a localidade, demonstrando a plasticidade que essa espécie possui para ocupar diferentes ambientes. As diferenças entre os ambientes estudados são, principalmente, a presença de linhas recifais e a influência estuarina.

Os valores da densidade de hastes encontrados nas populações de Alagoas foram relativamente inferiores a alguns estudos (GALLEGOS et al., 1994; CREED, 1999; HAUXWELL et al., 2001), mas superiores a outros (ELEUTERIUS, 1987; DE BOER, 2000; MAGALHÃES ET AL. 2003); KOWALSKI; DEYOE; ALLISON, 2009; SORDO et al., 2011). Locais com densidades menores são áreas onde se verifica grandes impactos naturais (como furacões) e antropogênicos (complexos portuários e dragagens), o que não é observado na área estudada.

Em relação aos comprimentos foliares de $H$. wrightii, as medidas das plantas de Alagoas foram inferiores aquelas encontrados por Magalhães, Eskinazi-Leça e Moura Júnior (1997) na Ilha de Itamaracá, norte de Pernambuco, Brasil, e por Pangallo e Bell (1988), que encontraram folhas com até $40 \mathrm{~cm}$ em Tampa Bay, Flórida. Porém Creed (1997) registrou comprimentos foliares semelhantes aos do presente estudo em praias do Rio de Janeiro, confirmando a plasticidade da espécie. Phillips (1960 e 1967), ao realizar estudos em diferentes lugares da Flórida, observou que as plantas seguiam um padrão de acordo com a localização dos prados: quanto mais próximas à costa, menores as folhas, que aumentam de tamanho à medida que se afastavam da costa. Entretanto Cabello-Pasini, Muñiz-Salazar e Ward (2003), sugerem que o comprimento foliar é alterado em função de uma resposta aos níveis de penetração da luz, em que as plantas precisam alongar suas folhas para atingir à camada superior mais iluminada. No presente estudo, as maiores folhas foram encontradas na área com maior influência marinha e área mais profunda, o que sugere que a penetração da luz e a profundidade influenciam o comprimento das plantas.

A largura foliar de $H$. wrightii nas praias estudadas foi superior aos valores descritos em outros estudos (PHILLIPS, 1960 e 1967; PANGALLO; BELL, 1988; CREED, 1997; MAGALHÃES; ESKINAZI-LEÇA; MOURA JUNIOR, 1997). Quando comparados com De Boer (2000), que encontrou valores entre $1,3 \mathrm{~mm}$ e $2,2 \mathrm{~mm}$, os resultados podem ser considerados semelhantes $(1,6 \mathrm{~mm}$ a $2,1 \mathrm{~mm})$. A largura foliar também é influenciada pela posição dos prados, sendo as folhas mais estreitas encontradas próximo à costa e as mais largas nas áreas permanentemente submersas. Segundo Eleuterius (1987), este é um dos fatores de maior influência para o tamanho e a largura das folhas, visto que quanto mais próximas à costa, maior influência há da força das ondas e dos ventos, além de impactos humanos diretos e indiretos.

\section{CONCLUSÃO}

Os resultados aqui descritos contribuem para o início do conhecimento das angiospermas marinhas no estado de Alagoas, já que são os primeiros dados ecológicos publicados sobre a morfologia de Halodule wrightii para esse Estado nordestino. Eles confirmam a plasticidade da espécie e como estas plantas tem destaque na preservação e determinação da soltura de peixes-bois marinhos por ser seu alimento preferencial, são sugeridos estudos e monitoramentos que incluam os fatores abióticos relativos à qualidade da água e sua movimentação, especialmente dentro da área da APA Costa dos Corais.

\section{AGRADECIMENTOS}

Os autores agradecem à Fundação O Boticário pelo apoio na realização da pesquisa e a à Fundação Mamíferos Aquáticos pelo suporte técnico.

\section{REFERÊNCIAS}

CABELLO-PASINI, A.; MUÑIZ-SALAZAR, R.; WARD, D. H. Annual variations of biomass and photosynthesis in Zosteramarina at its southern end of distribution in the North Pacific. Aquatic Botany, v. 76, p. 31-47. 2003. 
FRANÇA, C. R. C. de et. al. Morfologia foliar e densidade de hastes de Halodule wrightii (Cymodoceaceae), no litoral de Alagoas, Brasil.

CORREIA, M. D.; SOVIERZOSKI, H.H. Gestão e desenvolvimento sustentável da zona costeira do estado de Alagoas, Brasil. Revista de Gestão Costeira Integrada, v. 8, n. 2, p. 25-45. 2008.

CREED, J. C. Distribution, seasonal abundance and shoot size of the seagrass Halodule wrightii near its southern limit at Rio de Janeiro state, Brazil. Aquatic Botany, v. 65, p. 47-58.1997.

CREED, J. C. Distribution, seasonal abundance and shoot size of the seagrass Halodule wrightii near its southern limit at Rio de Janeiro state, Brazil. Aquatic Botany, v. 65, p. 47-58.1999.

CREED, J. C.; MONTEIRO, R. L. C. An analyses of the phenotypic variation in the seagrass Halodule wrightii Aschers. Leandra, Rio de Janeiro, v. 15, p. 1-9.2000.

DE BOER, W. F. Biomass dynamics of seagrassess and the role of mangrove and seagrass vegetation as different nutrient sources for an intertidal ecosystem. Aquatic Botany, v. 66, p. 225-239.2000.

DUARTE, C. M.; KIRKMAN, H. Methods for the measurement of seagrass abundance and depth distribution. In: SHORT, F.; COLES, R. (Eds.). Global Seagrass Research Methods, New York: Elsevier, 2001. p. 141-154.

DUNTON, K. H. Seasonal growth and biomass of the subtropical seagrass Halodule wrightii in relation to continuous measurements of underwater irradiance. Marine Biology, v. 120, p. 479-489. 1994.

ELEUTERIUS, L. N. Seagrass ecology along the coasts of Alabama, Louisiana, and Mississippi. In: DURAKO, M. J.; PHILLIPS, R. C.; LEWIS III, R. R. (Eds.).Proceedings of the Symposium on Subtropical-Tropical Seagrasses in the Southeastern United States. Florida: Florida Marine Research Publications, 1987. v. 42, p. 11-20.

ERFTEMEIJER, P. L. A.; HERMAN, P. M. J. Seasonal changes in environmental variables, biomass, production and nutrient contents in two contrasting tropical intertidal seagrass beds in South Sulawesi, Indonesia. Oecologia, v. 99, p. 45-59.1994.

GALLEGOS, M. E. et al. Growth patterns and demography of pioneer Caribbean seagrasses Halodule wrightii and Syringodium filiforme. Marine Ecology Progress Series, v. 109, p. 99104.1994.

GRANGER, S.; LIZUMI, H. Water quality measurement methods for seagrass habitat. In: SHORT, F.; COLES, R. (Eds.). Global Seagrass Research Methods, New York: Elsevier, 2001. p. 393-406.

HAUXWELL, J. et al. Measuring production of Halodulewrightii: additional evidence suggests clipping underestimates growth rate. Aquatic Botany, v. 69, p. 41-54.2001.

HEMMINGA, M. A.; HARRISON, P. G.; VAN LENT, F. The Balance of nutrient losses and gains in seagrass meadows. Marine Ecology Progress Series, v. 71, p. 85-96.1991. HEMMINGA, M. A.; DUARTE. C. M. Seagrass ecology. Cambridge: Cambridge University Press,2000. 298 p.

HOUSTON, A. I.; MCNAMARA, J. M. Phenotypic plasticity as a state-dependent life-history decision. Evolutionary Ecology, v. 6, p. 243-253. 1992.

$\mathrm{KOCH}, \mathrm{E}$. W. Beyond light: physical, geological and geochemical parameters as possible submersed aquatic vegetation habitat requeriments. Estuaries, v. 24, n. 1, p. 1-17. 2001.

KOWALSK, J. L.; DEYOE, H. R.; ALLISON, T. C. Seasonal Production and Biomass of the Seagrass, Halodule wrightii Aschers. (Shoal Grass), in a Subtropical Texas Lagoon. Estuaries and Coasts, v. 32, p. 467-482. 2009.

LABOREL-DEGUEN, F. Nota preliminar sobre a ecologia das pradarias de fanerógamas marinhas nas costas dos estados de Pernambuco e Paraíba. Trabalhos do Instituto de Biologia

Marítima e Oceanografia, v. 3, n.4, p. 39-50. 1963. 
FRANÇA, C. R. C. de et. al. Morfologia foliar e densidade de hastes de Halodule wrightii (Cymodoceaceae), no litoral de Alagoas, Brasil.

LEE, K. S.; DUNTON, K. H. Production and carbon reservedynamics of the seagrassThalassiatestudinumin Corpus ChristiBay, Texas, USA. Marine Ecology Progress Series, v. 143, p. 201-210. 1996.

MAGALHÃES, K. M.; ALVES, M. S. Fanerógamas marinhas do litoral do estado de Pernambuco. In: TABARELLI, M; DA SILVA, J. M. C. (Eds.). Diagnóstico da biodiversidade de Pernambuco. Recife: Massangana, 2002. v. 1, p. 173-182.

MAGALHÃES, K. M; ESKINAZI-LEÇA, E; JUNIOR, A. M. M. Morfometria e biomassa da fanerógama marinha Halodule wrightii Ascherson no litoral norte de Pernambuco. Trabalhos Oceanográficos da UFPE, v. 25, p. 83-92. 1997.

MAGALHÃES, K. M., COCENTINO, A. M., ESKINAZI-LEÇA, E.; FERNANDES, M. B.; REIS, T. N. V.; GUIMARÃES, N. C. L.; RODRIGES, H.S. Seagrass meadows at the Suape Port área, Pernambuco In: VI Congresso de Ecologia do Brasil, 2003, Fortaleza. Anais de trabalhos completos. Editora da Universidade Federal do Ceará, p.334-335. 2003.

OLIVEIRA FILHO, E. C.; PIRANI, J. R.; GUILIETTI, A. M. The brazilian seagrasses. Aquatic Botany, v. 56, p. 25-33, 1983.

OLIVEIRA-FILHO, E. C. et al. Phenology of a seagrass (Halodule wrightii) bed on the southeast coast of Brazil. Aquatic Botany, v. 56, p. 25-33.1997.

PANGALLO, R. A.; BELL, S. S. Dynamics of the aboveground and belowground structure of the seagrass Halodulewrightii. Marine Ecology Progress Series, v. 43, p. 297-301. 1988.

PÉREZ-LLORENS, J. L.; NIELL, F. X. Short-term phosphate uptake kinetics in: Zostera noltii Hornem: a comparison between excised leaves and sediment-rooted plants. Hydrobiologia, $v$. 297, n. 1, p. 17-27. 1995.

PHILLIPS, R. C. Environmental effect on leaves of Diplanthera Du Petit-Thouars. Bulletin Marine Science of the Gulf and Caribbean, v. 10, p. 346-353.1960.

PHILLIPS, R. C. On the species of the seagrass, Halodule, in Florida. Bulletin of Marine Science, v. 17, p. 672-676. 1967.

PHILLIPS, R. C. The seagrass ecosystem and resources in Latin America. In:U. SEELIGER (Ed.). Coastal plant communities of Latin America. New-York: Academic Press, 1992.p. 107-121.

SHORT, F. et al. Global seagrass distribution and diversity: A bioregional model. Journal Experimental Marine Biology and Ecology, 350, 3-20.

SORDO, L. et al. 2011. Temporal variations in morphology and biomass of vulnerable Halodule wrightii meadows at their southernmost distribution limit in the southwestern Atlantic.

Botanica Marina, v. 54, p. 136-21. 2007 